\title{
Tangential Motion of Fluids near Charged Surfaces: Consequences for Wetting
}

\author{
J. Lyklema' \\ 1 Department of Physical and Colloid Chemistry, Landbouw Universiteit Wageningen, \\ Dreijenplein 6, 6703 HB Wageningen - The Netherlands \\ e-mail: hans@fenk.wau.nl
}

Résumé - Déplacement tangentiel de fluides au voisinage de surfaces chargées : conséquences pour la mouillabilité — Les phénomènes d'électrocinétique font tous référence aux déplacements tangentiels des fluides par rapport aux surfaces solides chargées, ou réciproquement.

Nous présentons dans cet article une étude détaillée basée sur des mesures expérimentales de conduction de surface ainsi que sur des simulations de dynamique moléculaire. Ces travaux apportent un éclairage nouveau sur le phénomène de frottement, éclairage qui peut s'avérer pertinent pour notre compréhension de la dynamique de mouillage.

Mots-clés : électrocinétique, conduction de surface, dynamique de mouillage, couches stagnantes, viscosité de surface.

\footnotetext{
Abstract - Tangential Motion of Fluids near Charged Surfaces: Consequences for Wetting Electrokinetic phenomena all involve tangential motion of fluids with respect to charged solid surfaces or the other way around.

A penetrating study involving measurements of surface conduction and molecular dynamics yields mechanistic insight into the slip process which can be useful in interpreting wetting dynamics.

Keywords: electrokinetics, surface conduction, wetting dynamics, stagnant layers, surface viscosity.
} 


\section{INTRODUCTION}

Wetting dynamics and electrokinetics have at least one feature in common, viz. the issue of slip which may, or may not, occur if liquids flow tangentially to solid surfaces. The issue is directly related to the molecular density distribution $\rho(z)$ of the liquid adjacent to the solid, which is considered to be inert and hard.

Consider a drop of a pure, low molecular weight liquid $L$ partially wetting the solid $S$. General experience indicates that at the $L G$ (liquid/gas) phase boundary the $\rho(z)$ profile has a tanh-profile; over a few molecular layers the density decays from its bulk value to that in the vapour phase $G$. This follows from van der Waals theory, Cahn Hilliard theory and molecular dynamics (MD) simulations (see Lyklema, 2000 for a review). For a pure surface, there is no surface excess viscosity $\left(\eta^{\sigma}=0\right)$ (Langevin and Meunier, 1994; Earnshaw, 1996; Lucassen-Reynders and Lucassen, 1969). This follows both from surface rheology and surface light scattering so the statement is well-documented. For the present purpose the consequence is that, in treating the fluid dynamics of wetting, there is no need to consider any variation in the bulk fluidity near the $L G$ border. Far from the three-phase border, the interfacial tension $\gamma^{S L}$ at the solid-liquid interface, and the surface tension $\gamma^{L G}$ for the liquid surface against vapour can be related to the molecular details of the corresponding interface. For instance, Kirkwood and Buff (1949) interpreted $\gamma^{S L}$ in terms of distribution functions and for $L G$ interfaces $\gamma^{L G}$ is according to van der Waals and Cahn Hilliard related to the square of the density gradient, integrated over the entire transition layer. These two tensions, together with $\gamma^{S G}$, determine the contact angle. On the other hand, the overlap of the two differing liquid profiles at the $S L$ and $L G$ interfaces, which has to take place near the threephase border, gives rise to an additional Gibbs energy per unit length, which is responsible for the line tension.

We now focus on the $S L$ interface, emphasising the issue of slip upon an imposed tangential motion. In doing so we hope to contribute to the dynamics of spreading.

\section{INFORMATION FROM ELECTROKINETICS}

A large number of electrokinetic phenomena exist, some more familiar than others. Most well-known is electrophoresis, i.e. the moving of charged colloidal particles under the influence of an applied electric field. Other electrokinetic phenomena include electro-osmosis (movement of liquids, containing excess charges, through pores under an applied field), streaming potentials (creation of an electric field across pores by an applied pressure difference) and dielectric spectroscopy (frequency dependence of the polarisation of double layers around particles). All these phenomena involve tangential motion of a liquid (usually an aqueous electrolyte solution) with respect to a charged solid. In electrophoresis the liquid is stationary whereas the particles move; in the other phenomena mentioned the liquid moves with respect to the particles, to assemblies of particles in a porous plug, on the (charged) pores.

Electrokinetic phenomena cannot be interpreted without considering the notions of slip and stagnancy. This follows from the general observation that electrokinetic charge densities $\sigma^{e k}$ and electrokinetic (or zeta) potentials $\zeta$ usually differ from the corresponding surface charge densities $\sigma^{0}$ or potentials $\psi^{0}$, respectively. These differences are currently attributed to the existence of a thin stagnant layer of liquid which, upon the tangential movement, remains adhered to the solid surface. As these stagnant layers may contain a large part of the countercharge, the implication is that only a relatively small fraction of the double layer charge is electrokinetically active. The inactive part resides in the stagnant layer. As this is a relatively large fraction, electrokinetics is particularly suited for studying the properties of the layer.

Let us, as a next step, summarise the experience obtained over the past decades regarding these properties:

- the stagnant layer is only a few molecular layers thick. This follows from combining double layer studies with electrokinetics;

- the stagnant layer more on less coincides with the nondiffuse part of the double layer, i.e. with the Stern layer. It follows that for most practical purposes $\zeta$ may be identified with the potential of the diffuse part of the double layer $\psi^{d}$. For the interpretation of colloidal interaction this is of prime relevance, because the overlap of double layers is dominated by the diffuse double layer parts, and in practice the $\zeta$ potential is sometimes the only electric parameter available. For the present purpose it is relevant that $\sigma^{e k}$ may be computed from $\zeta$ using diffuse double layer theory, and as $\sigma^{0}$ is often obtainable by other means (for instance, by titration), the charge $\sigma^{i}$ in the stagnant layer can be evaluated because, as a whole, the double layer is electroneutral;

- stagnant layers are observed on hydrophilic and on hydrophobic surfaces. So, there is no reason to interpret them in terms of surface-liquid interaction. Rather, they are caused by stacking of the fluid molecules adjacent to the solid wall;

- on amphoteric surfaces, stagnant layers occur both on the negative and positive sides of the point of zero charge. There is no reason to assume them to be absent on uncharged surfaces. Only then, their existence cannot be established electrokinetically;

- in media of low dielectric permittivity the potential decays very slowly with distance; therefore $\sigma^{0}$ and $\sigma^{e k}$ are almost identical. Hence, in such systems one cannot obtain information on the stagnant layer by electrokinetic methods. 
Summarising, stagnant layers are general features of solidliquid interfaces. Most likely, they are caused by the oscillatory stacking of these molecules against the hard wall.

The next question is: what are the dynamics of such layers upon tangential motion? Again, electrokinetics is helpful because it is possible to obtain experimental information on the lateral mobility of ions in these layers by closer analysis of the phenomenon of surface conduction.

\section{CONDUCTION IN STAGNANT LAYERS}

The excess charge on the solution side of electric double layers gives rise to an excess tangential conductivity $K^{\sigma}$. The SI units are $\mathrm{S}=\mathrm{CV}^{-1} \mathrm{~s}^{-1}$. Surface conduction quantitatively affects all electrokinetic phenomena, to an extent determined by its ratio to the bulk conductivity $K^{L}$ (in $\mathrm{Sm}^{-1}=\mathrm{CV}^{-1} \mathrm{~m}^{-1} \mathrm{~s}^{-1}$ ). This ratio can be quantified through the Dukhin number $\mathrm{Du}$ :

$$
D u=K^{\sigma} / a K^{L}
$$

where $a$ is a characteristic length relating to the curvature of the surface: for colloidal spheres it is the particle radius and for a cylinder or a pore it is the inner radius. In principle, $K^{\sigma}$ is measurable, but some scrutiny is needed because we are dealing with an excess quantity. Let us first consider a simple illustration of such a measurement, which already shows some of the main features.

For the streaming potential $E_{\text {str }}$ under the influence of a pressure $\Delta P$ applied across a capillary of which the walls carry an electric double layer, characterised by an electrokinetic potential $\zeta$, one can derive (Lyklema, 1995):

$$
E_{\mathrm{str}}=\frac{\varepsilon_{0} \varepsilon \zeta \Delta P}{\eta\left(K^{L}+2 K^{\sigma} / a\right)}=\frac{\varepsilon_{0} \varepsilon \zeta \Delta P}{\eta K^{L}(1+2 D u)}
$$

where $\varepsilon_{0} \varepsilon$ is the dielectric permittivity of the liquid (which enters through the Poisson equation) and $\eta$ the viscosity of the liquid, accounting for the hydrodynamic resistance. The conduction enters the denominator because it accounts for the extent to which the built-up potential difference leaks away. Leakage takes place through the bulk and along the surface; the corresponding fractions are characterised by $K^{L}$ and $K^{\sigma}$, respectively. Equation (2) has two unknowns, $\zeta$ and $K^{\sigma}$, which can be obtained if $E_{\mathrm{slr}}$ is measured as a function of radius. A plot of $\varepsilon_{0} \varepsilon \Delta P / E_{\mathrm{slr}} \eta$ as a function of $a^{-1}$ yields the intercept $K^{L} / \zeta$ and the slope $2 K^{\sigma} / \zeta$. This is one of the ways for obtaining $K^{\sigma}$.

Two other procedures have been proposed by Kijlstra et al. (1992) and by Minor et al. (1998). The Kijlstra method is based on the fact that neglection of surface conduction leads in some electrokinetic techniques to an overestimation of $\zeta$, in others to an underestimation. For instance, ignoring $K^{\sigma}$ in Equation (2) underestimates $\zeta$; for dielectric spectroscopy it is the other way around. By carrying out different electrokinetic techniques on one and the same sample, $K^{\sigma}$ was found as that parameter for which the resulting $\zeta$ potentials coincided. Minor et al. (1998) measured the conduction of plugs as a function of the electrolyte concentration $c_{e l}$. Such plots are linear; for $c_{e l} \rightarrow 0$ an intercept is found, equal to $K^{\sigma}$.

The surface conduction consists of two parts, one caused by the diffuse part of the double layer, $K^{\sigma d}$, the other $K^{\sigma_{i}}$ resulting from the stagnant layer, which is the quantity in which we are now interested. Assuming additivity:

$$
K^{\sigma}=K^{\sigma d}+K^{\sigma_{i}}
$$

For $K^{\sigma d}$ a rigorous expression (by Bikerman, see Lyklema, $1995)$ is available. In the diffuse part, the ion concentrations are known at any position and their mobility may be equated to that in bulk. $K^{\sigma d}$ depends on $c_{e l}$ and $\zeta$ and also contains an electro-osmotic contribution. So, if $K^{\sigma d}$ is subtracted from $K^{\sigma}, K^{\sigma_{i}}$ is obtainable. If $\sigma^{i}$ is also known, as explained in Section 1 , the mobility $u_{i}^{i}$ of the ions of type $i$ in the stagnant layer is also obtainable, using:

$$
K^{\sigma i}=\sigma^{i} u_{i}^{i}
$$

assuming only one ionic species to be present in that layer.

As will be shown below, for many ions $u_{i}^{i} \neq 0$. At first sight, this is surprising: the layer is stagnant, which means that its viscosity is virtually infinitely high. Why would then ions embedded in it be mobile whereas the water is immobilised?

Looking for other literature examples where ions can move in a fixed fluid medium, gels come to mind. A few percent of gelatine can completely immobilise the water into which it is dissolved. However, the conductivity of electrolytes, dissolved in it, is hardly impeded as compared to bulk water. Neither is the diffusion coefficient of dissolved molecules hardly lowered in solutions by gelation. So, the observation is not unique.

In Table 1 some recent results are collected on the ratio $u_{i}^{i} / u_{i}^{L}$ between the ion mobility in the stagnant layer and that in the bulk.

These data are a collage of results obtained by $\mathrm{PhD}$ students and post docs in our department (J. Kijlstra, M. Minor and A. van der Linde, A. van der Wal, M. Löbbus and R. Barchini) that we will not discuss here in more detail. For the sake of comparison the data marked by an asterisk have been obtained by Verbich et al. (1999), using a very different method based on the difference between the isoelectric point and the isoconduction point. The following is observed:

- for monovalent counterions, the ratio is about unity, or at least not far below it. In fact, for all those instances where it is clearly below unity there are physico-chemical reasons to account for that: Monosphere is porous, adsorbed polymer layers or biological maze structures force tangentially moving ions to take a detour, and $\mathrm{Cl}^{-}$ions adsorb specifically to haematite. So the conclusion is that the stagnancy of the layer does not significantly impede the mobility; 
TABLE 1

Survey of ionic mobility ratios $u_{i}^{i} / u^{L}{ }_{i}$ between the stagnant layer and the bulk (various techniques and elaborations)

\begin{tabular}{l|c|c}
\hline System & Type of ion & $u_{i}^{i} / u_{i}^{L}$ \\
\hline Silica (Stöber) & $\mathrm{K}^{+}$ & 0.96 \\
Silica (Monosphere-1000, Merck) & $\mathrm{K}^{+}$ & 0.7 \\
& $\mathrm{Mg}^{2+}$ & 0.06 \\
Haematite & $\mathrm{Cl}^{-}$ & 0.7 \\
Poly (styrene) latex & $\mathrm{H}^{+}, \mathrm{Li}^{+}, \mathrm{Na}^{+}, \mathrm{K}^{+}$ & 0.85 \\
Ibid., plus adsorbed & $\mathrm{Ibid}^{+}$ & 0.60 \\
poly (ethylene oxide) layer & & \\
Liposome vesicles & $\mathrm{Na}^{+}$ & $\sim 1.0$ \\
Liposome vesicles & $\mathrm{Cs}^{+}$ & $\sim 1.0^{*}$ \\
Liposome vesicles & $\mathrm{Ca}^{2+}, \mathrm{Cd}^{2+}$ & \\
Liposome vesicles & $\mathrm{Cu}^{2+}$ & $\sim 0.6$ \\
Liposome vesicles & $\mathrm{Ca}^{2+}$ & $\sim 0.8^{*}$ \\
Liposome vesicles & $\mathrm{La}^{3+}$ & $\sim 0.07^{*}$ \\
Bacterial surfaces & $\mathrm{Na}^{+}$ & $0.2-0.5^{* *}$ \\
\hline
\end{tabular}

* Data from Verbich et al. (1999).

** Depending on species.

- the ratio decreases markedly if the valancy is increased, that is: when the attraction between counterion and surface is increased;

- at given ion valancy, there is no marked specificity. In particular it is noted that in the bulk protons are much more rapidly transferred than the other cations, because the mechanism is entirely different. Nevertheless, the mobility ratios do not differ measurably from those for the alkali metal ions.

Generally speaking, it may be concluded that we are primarily facing a generic phenomenon, onto which some secondary specificity is superimposed.

The question remains what the molecular interpretation is for these phenomena.

\section{MOLECULAR INTERPRETATION OF THE TANGENTIAL TRANSPORT}

An important step forward could be made by MD simulations (Lyklema et al., 1998). This development is still in its embryonic state. So far only Lennard-Jones interactions have been considered, which is a far cry from electric double layers. However, because of the genericity of the phenomena, already on this level useful new physical insight was obtainable. We refer to Lyklema et al. (1998) for numerical details.

Basically, a liquid in contact with a solid was simulated, with inside the liquid a number of molecules ("ions") that were stronger attracted by the solid than the liquid molecules. A certain distribution $\rho(z)$ developed for both the liquid molecules and the dissolved ions. Then a tangential force was applied on the ions. Bulk ions undergo a hectic stochastic thermal motion, onto which the movement in the direction of the electric field is superimposed. This represents bulk conduction. Ions adjacent to the surface in the stagnant layer remain part of the time in this layer, also moving in the direction of the applied force, but they also can make excursions to the bulk, from which they can return or where they may remain. In the latter case, another bulk ion returns to the stagnant layer to replenish this layer and keep the distribution intact. So, the tangential motion in the stagnant layer is, at least in part, short-circuited through the bulk. Hence, $u_{i}^{i}$ is an average of stagnant layer and bulk mobility weighted according to the fraction of the time that the ions reside in these two states. Resolving these fractions is still under study. However, it can be seen how the valence influence enters: for higher charged counterions the probability of desorption is less, so motion in the stagnant layer and short-circuiting are both impeded. The parameters selected for the various Lennard-Jones pair interactions in Lyklema et al. (1998) were adequate to confirm our experimental finding that for monovalent ions $u_{i}^{i} / u_{i}^{L}$ is very close to unity.

Regarding the solvent (water), two features were recovered. First, the trajectories of water molecules did not differ greatly from those of the ions, even though no force was exerted on the solvent. This finding is nothing else than simulation of electro-osmosis. It implies that on the individual scale water molecules in the stagnant layer are mobile with a mobility close to that in bulk.

The question remains why stagnant layers behave macroscopically as if they have a high viscosity. From the simulations the viscosity can also be determined (from the time correlation function). It was found that in the stagnant layer $\eta$ is substantially higher than in the bulk and anisotropic. Viscosity represents the resistance against collective motion of large sets of molecules. The adjoining fixed wall thwarts this collectivity. At the same time, this difference between individual and collective motion also accounts for the unimpeded diffusion of dissolved molecules in three-dimensional gels.

We hope to pursue this promising development which captures most experimental findings.

\section{CONCLUSION}

One of the central issues in wetting dynamics is that of slip at the solid-liquid phase boundary. Similar slip phenomena also occur in electrokinetics. A wealth of information has been built up about the so-called stagnant layer adjacent to the solid. This layer is a few molecular diameters thick and has the properties of a two-dimensional gel: macroscopically it is immobilised but the mobility of monovalent ions in it is not markedly lower than in bulk. These features can be recovered by MD simulations. 


\section{REFERENCES}

Earnshaw, J.C. (1996) Light Scattering as a Probe of Liquid Surfaces and Interfaces. Adv. Colloid Interface Sci., 68, 1.

Kijlstra, J., Van Leeuwen, H.P. and Lyklema, J. (1992) Effects of Surface Conduction on the Electrokinetic Properties Colloids. J. Chem. Soc. Faraday Trans., 88, 3441-3449.

Kirkwood, J.C. and Buff, F.P. (1949) The Statistical Mechanical Theory of Surface Tension. J. Chem. Phys., 17, 338-343.

Langevin, D. and Meunier, J. (1994) Interfacial Tension; Theory and Experiment, in Micelles, Membranes, Microemulsions and Monolayers, Gelbart, W.N., Ben-Shaul, A. and Roux, D. (eds.), Chap. 10, Springer.

Lucassen-Reynders, E.H. and Lucassen, J. (1969) Properties of Capillary Waves. Adv. Colloid Interface Sci., 2, 347-395.

Lyklema, J. (1995) Electrokinetics and Related Phenomena, in Fundamentals of Interface and Colloid Science, Vol. II, Chap. 4, Academic Press.
Lyklema, J. (2000) Surface Tensions: Interpretation, in Fundamentals of Interface and Colloid Science, Vol. III, Chap. 2, Academic Press.

Lyklema, J., Rovillard, S. and De Coninck, J. (1998) Electrokinetics: The Properties of the Stagnant Layer Unraveled. Langmuir, 14, 5659-5663.

Minor, M., van der Linde, A.J. and Lyklema, J. (1998) Streaming Potentials and Conductivities of Porous Silica Plugs. Colloids Surf., A142, 165-173.

Verbich, S.V., Dukhin, S.S. and Matsumura, H. (1999) Investigation of Dynamic Stern Layer of Liposomes by Measurements of Conductivity and Electrophoresis. J. Disp. Sci. Technol., 20, 83-104.

Final manuscript received in November 2000 